\title{
DYNAMICS OF QCD IN A STRONG MAGNETIC FIELD
}

\author{
V.A. MIRANSKY* \\ Department of Applied Mathematics, \\ University of Western Ontario, \\ London, Ontario N6A 5B\%, Canada
}

QCD in a strong magnetic field yields an example of a rich, sophisticated and controllable dynamics.

\section{Prologue}

On the first day of this wonderful meeting, I decided to change the topic of my talk. Already during the first Session, I felt it necessary to present something special, something connected with Arkady. So, I decided to talk about the dynamics of QCD in a strong magnetic field. This talk, it seemed to me, was appropriate indeed. The point is that the last time when I interacted with Arkady in person was in April 1994, in the Institute for Theoretical Physics in Santa Barbara. At that time, we, Valery Gusynin, Igor Shovkovy, and myself, had just finished our first work [1] in the series of papers concerning the role of a magnetic field in dynamical symmetry breaking in $2+1$ and $3+1$ dimensional field theories (actually, at that time, we discussed only the $2+1$ dimensional case). So, it seemed to be appropriate to "continue" that discussion at the Arkadyfest in Minneapolis 8 years later.

I was lucky with this decision. At the time of its presentation, this work had not been yet completed. During my talk, Arkady raised a question concerning the dynamics of the magnetic catalysis in QCD with a large number of colors, $N_{c} \rightarrow \infty$. The answer to this question, Igor Shovkovy and I got the next day, helped to finish the work [2].

\section{Introduction}

Since the dynamics of QCD is extremely rich and complicated, it is important to study this theory under external conditions which provide a controllable dynamics. On the one hand, this allows one to understand better the vacuum structure and Green's functions of QCD, and, on the other hand, 
there can exist interesting applications of such models in themselves. The well known examples are hot QCD and QCD with a large baryon density.

Studies of QCD in external electromagnetic fields had started long ago $[3,4]$. A particularly interesting case is an external magnetic field. Using the Nambu-Jona-Lasinio (NJL) model as a low energy effective theory for QCD, it was shown that a magnetic field enhances the spontaneous chiral symmetry breakdown. The understanding of this phenomenon had remained obscure until a universal role of a magnetic field as a catalyst of chiral symmetry breaking was established in Refs. [1,5]. The general result states that a constant magnetic field leads to the generation of a fermion dynamical mass (i.e., a gap in the one-particle energy spectrum) even at the weakest attractive interaction between fermions. For this reason, this phenomenon was called the magnetic catalysis. The essence of the effect is the dimensional reduction $D \rightarrow D-2$ in the dynamics of fermion pairing in a magnetic field. In the particular case of weak coupling, this dynamics is dominated by the lowest Landau level (LLL) which is essentially $D-2$ dimensional $[1,5]$.

The phenomenon of the magnetic catalysis was studied in gauge theories, in particular, in QED [6,7] and in QCD [ 8,9]. In the recent work [9], it has been suggested that the dynamics underlying the magnetic catalysis in QCD is weakly coupled at sufficiently large magnetic fields. Here we will consider this dynamical problem rigorously, from first principles. In fact, we show that, at sufficiently strong magnetic fields, $|e B| \gg \Lambda_{Q C D}^{2}$, there exists a consistent truncation of the Schwinger-Dyson (gap) equation which leads to a reliable asymptotic expression for the quark mass $m_{q}$. Its explicit form reads:

$$
m_{q}^{2} \simeq 2 C_{1}\left|e_{q} B\right|\left(c_{q} \alpha_{s}\right)^{2 / 3} \exp \left[-\frac{4 N_{c} \pi}{\alpha_{s}\left(N_{c}^{2}-1\right) \ln \left(C_{2} / c_{q} \alpha_{s}\right)}\right]
$$

where $e_{q}$ is the electric charge of the $q$-th quark and $N_{c}$ is the number of colors. The numerical factors $C_{1}$ and $C_{2}$ equal 1 in the leading approximation that we use. Their value, however, can change beyond this approximation and we can only say that they are of order 1 . The constant $c_{q}$ is defined as follows:

$$
c_{q}=\frac{1}{6 \pi}\left(2 N_{u}+N_{d}\right)\left|\frac{e}{e_{q}}\right| \text {, }
$$

where $N_{u}$ and $N_{d}$ are the numbers of up and down quark flavors, respectively. The total number of quark flavors is $N_{f}=N_{u}+N_{d}$. The strong 
coupling $\alpha_{s}$ in the last equation is related to the scale $\sqrt{|e B|}$, i.e.,

$$
\frac{1}{\alpha_{s}} \simeq b \ln \frac{|e B|}{\Lambda_{Q C D}^{2}}, \quad \text { where } \quad b=\frac{11 N_{c}-2 N_{f}}{12 \pi}
$$

We should note that in the leading approximation the energy scale $\sqrt{|e B|}$ in Eq. (3) is fixed only up to a factor of order 1.

The central dynamical issue underlying this dynamics is the effect of screening of the gluon interactions in a magnetic field in the region of momenta relevant for the chiral symmetry breaking dynamics, $m_{q}^{2} \ll\left|k^{2}\right| \ll$ $|e B|$. In this region, gluons acquire a mass $M_{g}$ of order $\sqrt{N_{f} \alpha_{s}\left|e_{q} B\right|}$. This allows to separate the dynamics of the magnetic catalysis from that of confinement.

Since the background magnetic field breaks explicitly the global chiral symmetry that interchanges the up and down quark flavors, the chiral symmetry in this problem is $S U\left(N_{u}\right)_{L} \times S U\left(N_{u}\right)_{R} \times S U\left(N_{d}\right)_{L} \times S U\left(N_{d}\right)_{R} \times$ $U^{(-)}(1)_{A}$. The $U^{(-)}(1)_{A}$ is connected with the current which is an anomaly free linear combination of the $U^{(d)}(1)_{A}$ and $U^{(u)}(1)_{A}$ currents. The generation of quark masses breaks this symmetry spontaneously down to $S U\left(N_{u}\right)_{V} \times S U\left(N_{d}\right)_{V}$ and, as a result, $N_{u}^{2}+N_{d}^{2}-1$ gapless NambuGoldstone (NG) bosons occur. In Sec. 4, we derive the effective action for the NG bosons and calculate their decay constants and velocities.

The present analysis is heavily based on the analysis of the magnetic catalysis in QED done by Gusynin, Miransky, and Shovkovy [7]. A crucial difference is of course the property of asymptotic freedom and confinement in QCD. In connection with that, our second major result is the derivation of the low energy effective action for gluons in QCD in a strong magnetic field. The characteristic feature of this action is its anisotropic dynamics. In particular, the strength of static (Coulomb like) forces along the direction parallel to the magnetic field is much larger than that in the transverse directions. Also, the confinement scale in this theory is much less than that in QCD without a magnetic field. This features imply a rich and unusual spectrum of light glueballs in this theory.

A special and interesting case is QCD with a large number of colors, in particular, with $N_{c} \rightarrow \infty$ (the 't Hooft limit). The question about it was raised by Arkady during my talk. This theory is considered in Sec. 6 . 


\section{Magnetic catalysis in QCD}

We begin by considering the Schwinger-Dyson (gap) equation for the quark propagator. It has the following form:

$$
G^{-1}(x, y)=S^{-1}(x, y)+4 \pi \alpha_{s} \gamma^{\mu} \int G(x, z) \Gamma^{\nu}(z, y, u) D_{\nu \mu}(u, x) d^{4} z d^{4} u(4)
$$

where $S(x, y)$ and $G(x, y)$ are the bare and full fermion propagators in an external magnetic field, $D_{\nu \mu}(x, y)$ is the full gluon propagator and $\Gamma^{\nu}(x, y, z)$ is the full amputated vertex function. Since the coupling $\alpha_{s}$ related to the scale $|e B|$ is small, one might think that the rainbow (ladder) approximation is reliable in this problem. However, this is not the case. Because of the $(1+1)$-dimensional form of the fermion propagator in the LLL approximation, there are relevant higher order contributions [6,7]. Fortunately one can solve this problem. First of all, an important feature of the quarkantiquark pairing dynamics in QCD in a strong magnetic field is that this dynamics is essentially abelian. This feature is provided by the form of the polarization operator of gluons in this theory. The point is that the dynamics of the quark-antiquark pairing is mainly induced in the region of momenta $k$ much less than $\sqrt{|e B|}$. This implies that the magnetic field yields a dynamical ultraviolet cutoff in this problem. On the other hand, while the contribution of (electrically neutral) gluons and ghosts in the polarization operator is proportional to $k^{2}$, the fermion contribution is proportional to $\left|e_{q} B\right|[7]$. As a result, the fermion contribution dominates in the relevant region with $k^{2} \ll|e B|$.

This observation implies that there are three, dynamically very different, scale regions in this problem. The first one is the region with the energy scale above the magnetic scale $\sqrt{|e B|}$. In that region, the dynamics is essentially the same as in QCD without a magnetic field. In particular, the running coupling decreases logarithmically with increasing the energy scale there. The second region is that with the energy scale below the magnetic scale but much larger than the dynamical mass $m_{q}$. In this region, the dynamics is abelian like and, therefore, the dynamics of the magnetic catalysis is similar to that in QED in a magnetic field. At last, the third region is the region with the energy scale less than the gap. In this region, quarks decouple and a confinement dynamics for gluons is realized.

Let us first consider the intermediate region relevant for the magnetic catalysis. As was indicated above, the important ingredient of this dynamics is a large contribution of fermions to the polarization operator. It is large because of an (essentially) $1+1$ dimensional form of the fermion propagator in a strong magnetic field. Its explicit form can be obtained 
by modifying appropriately the expression for the polarization operator in QED in a magnetic field [7]:

$$
\begin{gathered}
P^{A B, \mu \nu} \simeq \frac{\alpha_{s}}{6 \pi} \delta^{A B}\left(k_{\|}^{\mu} k_{\|}^{\nu}-k_{\|}^{2} g_{\|}^{\mu \nu}\right) \sum_{q=1}^{N_{f}} \frac{\left|e_{q} B\right|}{m_{q}^{2}}, \quad\left|k_{\|}^{2}\right| \ll m_{q}^{2}, \\
P^{A B, \mu \nu} \simeq-\frac{\alpha_{s}}{\pi} \delta^{A B}\left(k_{\|}^{\mu} k_{\|}^{\nu}-k_{\|}^{2} g_{\|}^{\mu \nu}\right) \sum_{q=1}^{N_{f}} \frac{\left|e_{q} B\right|}{k_{\|}^{2}}, \quad m_{q}^{2} \ll\left|k_{\|}^{2}\right| \ll|e B|,
\end{gathered}
$$

where $g_{\|}^{\mu \nu} \equiv \operatorname{diag}(1,0,0,-1)$ is the projector onto the longitudinal subspace, and $k_{\|}^{\mu} \equiv g_{\|}^{\mu \nu} k_{\nu}$ (the magnetic field is in the $x^{3}$ direction). Similarly, we introduce the orthogonal projector $g_{\perp}^{\mu \nu} \equiv g^{\mu \nu}-g_{\|}^{\mu \nu}=\operatorname{diag}(0,-1,-1,0)$ and $k_{\perp}^{\mu} \equiv g_{\perp}^{\mu \nu} k_{\nu}$ that we shall use below. Notice that quarks in a strong magnetic field do not couple to the transverse subspace spanned by $g_{\perp}^{\mu \nu}$ and $k_{\perp}^{\mu}$. This is because in a strong magnetic field only the quark from the LLL matter and they couple only to the longitudinal components of the gluon field. The latter property follows from the fact that spins of the LLL quarks are polarized along the magnetic field (see the second paper in [5]).

The expressions (5) and (6) coincide with those for the polarization operator in the $1+1$ dimensional massive QED (massive Schwinger model) [10] if the parameter $\alpha_{s}\left|e_{q} B\right| / 2$ here is replaced by the dimensional coupling $\alpha_{1}$ of $Q E D_{1+1}$. As in the Schwinger model, Eq. (6) implies that there is a massive resonance in the $k_{\|}^{\mu} k_{\|}^{\nu}-k_{\|}^{2} g_{\|}^{\mu \nu}$ component of the gluon propagator. Its mass is

$$
M_{g}^{2}=\sum_{q=1}^{N_{f}} \frac{\alpha_{s}}{\pi}\left|e_{q} B\right|=\left(2 N_{u}+N_{d}\right) \frac{\alpha_{s}}{3 \pi}|e B| .
$$

This is reminiscent of the pseudo-Higgs effect in the $(1+1)$-dimensional massive QED. It is not the genuine Higgs effect because there is no complete screening of the color charge in the infrared region with $\left|k_{\|}^{2}\right| \ll m_{q}^{2}$. This can be seen clearly from Eq. (5). Nevertheless, the pseudo-Higgs effect is manifested in creating a massive resonance and this resonance provides the dominant forces leading to chiral symmetry breaking.

Now, after the abelian like structure of the dynamics in this problem is established, we can use the results of the analysis in QED in a magnetic field [7] by introducing appropriate modifications. The main points of the analysis are: (i) the so called improved rainbow approximation is reliable in this problem provided a special non-local gauge is used in the analysis, and (ii) for a small coupling $\alpha_{s}$ ( $\alpha$ in QED), the relevant region of momenta in this problem is $m_{q}^{2} \ll\left|k^{2}\right| \ll|e B|$. We recall that in the 
improved rainbow approximation the vertex $\Gamma^{\nu}(x, y, z)$ is taken to be bare and the gluon propagator is taken in the one-loop approximation. Moreover, as we argued above, in this intermediate region of momenta, only the contribution of quarks to the gluon polarization tensor (6) matters. [It is appropriate to call this approximation the "strong-field-loop improved rainbow approximation". It is an analog of the hard-dense-loop improved rainbow approximation in QCD with a nonzero baryon density]. As to the modifications, they are purely kinematic: the overall coupling constant in the gap equation $\alpha$ and the dimensionless combination $M_{\gamma}^{2} /|e B|$ in QED have to be replaced by $\alpha_{s}\left(N_{c}^{2}-1\right) / 2 N_{c}$ and $M_{g}^{2} /\left|e_{q} B\right|$, respectively. This leads us to the expression (1) for the dynamical gap.

After expressing the magnetic field in terms of the running coupling, the result for the dynamical mass takes the following convenient form:

$$
m_{q}^{2} \simeq 2 C_{1}\left|\frac{e_{q}}{e}\right| \Lambda_{Q C D}^{2}\left(c_{q} \alpha_{s}\right)^{2 / 3} \exp \left[\frac{1}{b \alpha_{s}}-\frac{4 N_{c} \pi}{\alpha_{s}\left(N_{c}^{2}-1\right) \ln \left(C_{2} / c_{q} \alpha_{s}\right)}\right] .
$$

As is easy to check, the dynamical mass of the $u$-quark is considerably larger than that of the $d$-quark. It is also noticeable that the values of the $u$-quark dynamical mass becomes comparable to the vacuum value $m_{d y n}^{(0)} \simeq 300 \mathrm{MeV}$ only when the coupling constant gets as small as 0.05 .

Now, by trading the coupling constant for the magnetic field scale $|e B|$, we get the dependence of the dynamical mass on the value of the external field. The numerical results are presented in Fig. 1 [we used $C_{1}=C_{2}=$ 1 in Eq. (8)]. As one can see in Fig. 1, the value of the quark gap in a wide window of strong magnetic fields, $\Lambda_{Q C D}^{2} \ll|e B| \lesssim(10 \mathrm{TeV})^{2}$, remains smaller than the dynamical mass of quarks $m_{d y n}^{(0)} \simeq 300 \mathrm{MeV}$ in QCD without a magnetic field. In other words, the chiral condensate is partially suppressed for those values of a magnetic field. The explanation of this, rather unexpected, result is actually simple. The magnetic field leads to the mass $M_{g}(7)$ for gluons. In a strong enough magnetic field, this mass becomes larger than the characteristic gap $\Lambda$ in QCD without a magnetic field $(\Lambda$, playing the role of a gluon mass, can be estimated as a few times larger than $\left.\Lambda_{Q C D}\right)$. This, along with the property of the asymptotic freedom (i.e., the fact that $\alpha_{s}$ decreases with increasing the magnetic field), leads to the suppression of the chiral condensate.

This point also explains why our result for the gap is so different from that in the NJL model in a magnetic field [3]. Recall that, in the NJL model, the gap logarithmically (i.e., much faster than in the present case) grows with a magnetic field. This is the related to the assumption that both the dimensional coupling constant $G=g / \Lambda^{2}$ (with $\Lambda$ playing a role similar 


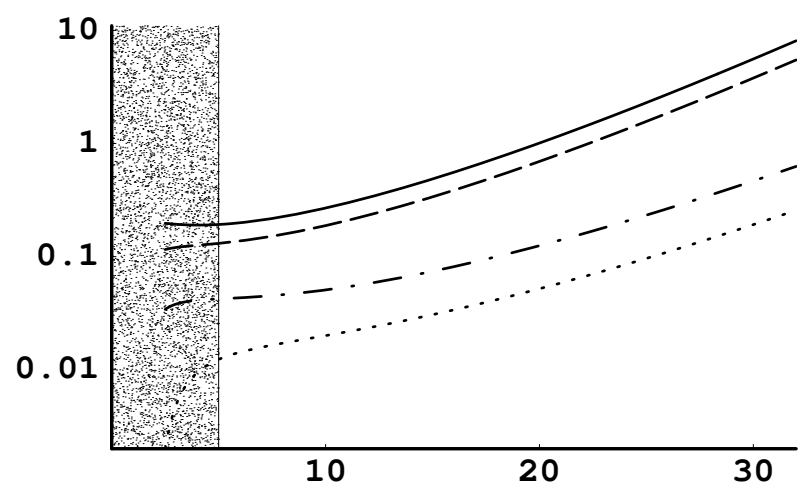

Figure 1. The dynamical masses of quarks as functions of $\ln \left(|e B| / \Lambda_{Q C D}^{2}\right)$ for $N_{c}=3$ and two different values of $N_{f}=N_{u}+N_{d}$ : (i) masses of $u$-quark (solid line) and $d$-quark (dash-dotted line) for $N_{u}=1$ and $N_{d}=2$; (ii) masses of $u$-quark (dashed line) and $d$ quark (dotted line) for $N_{u}=2$ and $N_{d}=2$. The result may not be reliable in the weak magnetic field region (shaded) where some of the approximations break. The values of masses are given in units of $\Lambda_{Q C D}=250 \mathrm{MeV}$.

to that of the gluon mass in QCD), as well as the scale $\Lambda$ do not dependent on the value of the magnetic field. Therefore, in that model, in a strong enough magnetic field, the value of the chiral condensate is overestimated.

The picture which emerges from this discussion is the following. For values of a magnetic field $|e B| \lesssim \Lambda^{2}$ the dynamics in QCD should be qualitatively similar to that in the NJL model. For strong values of the field, however, it is essentially different, as was described above. This in turn suggests that there should exist an intermediate region of fields where the dynamical masses of quarks decreases with increasing the background magnetic field.

\section{Effective action of NG bosons}

The presence of the background magnetic field breaks explicitly the global chiral symmetry that interchanges the up and down quark flavors. This is related to the fact that the electric charges of the two sets of quarks are different. However, the magnetic field does not break the global chiral symmetry of the action completely. In particular, in the model with the $N_{u}$ up quark flavors and the $N_{d}$ down quark flavors, the action is invariant under the chiral symmetry $S U\left(N_{u}\right)_{L} \times S U\left(N_{u}\right)_{R} \times S U\left(N_{d}\right)_{L} \times$ $S U\left(N_{d}\right)_{R} \times U^{(-)}(1)_{A}$. The $U^{(-)}(1)_{A}$ is connected with the current which is an anomaly free linear combination of the $U^{(d)}(1)_{A}$ and $U^{(u)}(1)_{A}$ currents. 
[The $U^{(-)}(1)_{A}$ symmetry is of course absent if either $N_{d}$ or $N_{u}$ is equal to zero].

The global chiral symmetry of the action is broken spontaneously down to the diagonal subgroup $S U\left(N_{u}\right)_{V} \times S U\left(N_{d}\right)_{V}$ when dynamical masses of quarks are generated. In agreement with the Goldstone theorem, this leads to the appearance of $N_{u}^{2}+N_{d}^{2}-1$ number of the NG gapless excitations in the low-energy spectrum of QCD in a strong magnetic field. Notice that there is also a pseudo-NG boson connected with the conventional (anomalous) $U(1)_{A}$ symmetry which can be rather light in a sufficiently strong magnetic field.

Now, in the chiral limit, the general structure of the low energy action for the NG bosons could be easily established from the symmetry arguments alone. First of all, such an action should be invariant with respect to the space-time symmetry $S O(1,1) \times S O(2)$ which is left unbroken by the background magnetic field [here the $\mathrm{SO}(1,1)$ and the $\mathrm{SO}(2)$ are connected with Lorentz boosts in the $x_{0}-x_{3}$ hyperplane and rotations in the $x_{1}-x_{2}$ plane, respectively]. Besides that, the low-energy action should respect the original chiral symmetry $S U\left(N_{u}\right)_{L} \times S U\left(N_{u}\right)_{R} \times S U\left(N_{d}\right)_{L} \times S U\left(N_{d}\right)_{R} \times$ $U^{(-)}(1)_{A}$. These requirements lead to the following general form of the action:

$$
\begin{aligned}
L_{N G} & \simeq \frac{f_{u}^{2}}{4} \operatorname{tr}\left(g_{\|}^{\mu \nu} \partial_{\mu} \Sigma_{u} \partial_{\nu} \Sigma_{u}^{\dagger}+v_{u}^{2} g_{\perp}^{\mu \nu} \partial_{\mu} \Sigma_{u} \partial_{\nu} \Sigma_{u}^{\dagger}\right) \\
& +\frac{f_{d}^{2}}{4} \operatorname{tr}\left(g_{\|}^{\mu \nu} \partial_{\mu} \Sigma_{d} \partial_{\nu} \Sigma_{d}^{\dagger}+v_{d}^{2} g_{\perp}^{\mu \nu} \partial_{\mu} \Sigma_{d} \partial_{\nu} \Sigma_{d}^{\dagger}\right) \\
& +\frac{\tilde{f}^{2}}{4}\left(g_{\|}^{\mu \nu} \partial_{\mu} \tilde{\Sigma} \partial_{\nu} \tilde{\Sigma}^{\dagger}+\tilde{v}^{2} g_{\perp}^{\mu \nu} \partial_{\mu} \tilde{\Sigma} \partial_{\nu} \tilde{\Sigma}^{\dagger}\right) .
\end{aligned}
$$

The unitary matrix fields $\Sigma_{u} \equiv \exp \left(i \sum_{A=1}^{N_{u}^{2}-1} \lambda^{A} \pi_{u}^{A} / f_{u}\right), \quad \Sigma_{d} \equiv$ $\exp \left(i \sum_{A=1}^{N_{d}^{2}-1} \lambda^{A} \pi_{d}^{A} / f_{d}\right)$, and $\tilde{\Sigma} \equiv \exp (i \sqrt{2} \tilde{\pi} / \tilde{f})$ describe the NG bosons in the up, down, and $U^{(-)}(1)_{A}$ sectors of the original theory. The decay constants $f_{u}, f_{d}, \tilde{f}$ and transverse velocities $v_{u}, v_{d}, \tilde{v}$ can be calculated by using the standard field theory formalism. The results for the $N_{u}^{2}+N_{d}^{2}-2$ NG bosons in the up and down sectors, assigned to the adjoint representation of the $S U\left(N_{u}\right)_{V} \times S U\left(N_{d}\right)_{V}$ symmetry, are [2]

$$
\begin{aligned}
f_{u}^{2} & =\frac{N_{c}}{6 \pi^{2}}|e B|, \\
f_{d}^{2} & =\frac{N_{c}}{12 \pi^{2}}|e B|, \quad v_{q}=0 .
\end{aligned}
$$

The remarkable fact is that the decay constants are nonzero even in the limit when the dynamical masses of quarks approach zero. The reason of 
that is the $1+1$ dimensional character of this dynamics. A similar situation takes place in color superconductivity: in that case the $1+1$ dimensional character of the dynamics is provided by the Fermi surface.

Notice that the transverse velocities of the NG bosons are equal to zero. This is also a consequence of the $1+1$ dimensional structure of the quark propagator in the LLL approximation. The point is that quarks can move in the transverse directions only by hopping to higher Landau levels. Taking into account higher Landau levels would lead to nonzero velocities suppressed by powers of $\left|m_{q}\right|^{2} /|e B|$. In fact, the explicit form of the velocities was derived in the weakly coupled NJL model in an external magnetic field [see Eq. (65) in the second paper of Ref. [5]]. It is

$$
v_{u, d}^{2} \sim \frac{\left|m_{u, d}\right|^{2}}{|e B|} \ln \frac{|e B|}{\left|m_{u, d}\right|^{2}} \ll 1 .
$$

A similar expression should take place also for the transverse velocities of the NG bosons in QCD.

The decay constant $\tilde{f}$ of the singlet NG boson connected with the spontaneous breakdown of the $U^{(-)}(1)_{A}$ is [2]

$$
\tilde{f}^{2}=\frac{\left(N_{d} f_{u}+N_{u} f_{d}\right)^{2}}{N_{f}^{2}}=\frac{\left(\sqrt{2} N_{d}+N_{u}\right)^{2} N_{c}}{12 \pi^{2} N_{f}^{2}}|e B| .
$$

Its transverse velocity is of course zero in the LLL approximation.

\section{Anisotropic confinement of gluons}

Let us now turn to the infrared region with $|k| \lesssim m_{d}$, where all quarks decouple (notice that we take here the smaller mass of $d$ quarks). In that region, a pure gluodynamics realizes. However, its dynamics is quite unusual. The point is that although gluons are electrically neutral, their dynamics is strongly influenced by an external magnetic field, as one can see from expression (5) for their polarization operator. In a more formal language, while quarks decouple and do not contribute into the equations of the renormalization group in that infrared region, their dynamics strongly influence the boundary (matching) conditions for those equations at $k \sim m_{d}$. A conventional way to describe this dynamics is the method of the low energy effective action. This low effective action was derived in Ref. [2]. Here we will discuss its main properties.

The low energy effective action is relevant for momenta $|k| \lesssim m_{d}$. Notice the following important feature of the action: its "bare" coupling constant $g$, related to the scale $m_{d}$, coincides with the value of the vacuum QCD coupling related to the scale $\sqrt{|e B|}$ (and not to the scale $m_{d}$ ). This is 
because $g$ is determined from the matching condition at $|k| \sim m_{d}$, the lower border of the intermediate region $m_{d} \lesssim|k| \lesssim \sqrt{|e B|}$, where, because of the pseudo-Higgs effect, the running of the coupling is essentially frozen. Therefore the "bare" coupling $g$ indeed coincides with the value of the vacuum QCD coupling related to the scale $\sqrt{|e B|}: g=g_{s}$. Since this value is much less that that of the vacuum QCD coupling related to the scale $m_{d}$, this implies that the confinement scale $\lambda_{Q C D}$ of the action should be much less than $\Lambda_{Q C D}$ in QCD without a magnetic field.

Actually, this consideration somewhat simplifies the real situation. Since the LLL quarks couple to the longitudinal components of the polarization operator, only the effective coupling connected with longitudinal gluons is frozen. For transverse gluons, there should be a logarithmic running of their effective coupling. It is clear, however, that this running should be quite different from that in the vacuum QCD. The point is that the time like gluons are now massive and their contribution in the running in the intermediate region is severely reduced. On the other hand, because of their negative norm, just the time like gluons are the major players in producing the antiscreening running in QCD (at least in covariant gauges). Since now they effectively decouple, the running of the effective coupling for the transverse gluons should slow down. It is even not inconceivable that the antiscreening running can be transformed into a screening one. In any case, one should expect that the value of the transverse coupling related to the matching scale $m_{d}$ will be also essentially reduced in comparison with that in the vacuum QCD. Since the consideration in this section is rather qualitative, we adopt the simplest scenario with the value of the transverse coupling at the matching scale $m_{d}$ also coinciding with $g_{s}$.

The interaction potential between two static quarks in this theory at "short" distances $r \sim m_{d}^{-1}$ reads:

$$
V(x, y, z) \simeq \frac{g_{s}^{2}}{4 \pi \sqrt{z^{2}+\epsilon\left(x^{2}+y^{2}\right)}},
$$

where the dielectric constant $\epsilon=1+\frac{\alpha_{s}}{6 \pi} \sum_{q=1}^{N_{f}}\left|e_{q} B\right| / m_{q}^{2}$ is very large. Because of the dielectric constant, this Coulomb like interaction is anisotropic in space: it is suppressed by a factor of $\sqrt{\epsilon}$ in the transverse directions compared to the interaction in the direction of the magnetic field.

The potential (14) corresponds to the classical, tree, approximation which is good only in the region of distances much smaller than the confinement radius $r_{Q C D} \sim \lambda_{Q C D}^{-1}$. Deviations from this interaction are determined by loop corrections. The analysis of the loop expansion leads to the following estimate of the new confinement scale $\lambda_{Q C D}$ in QCD in a strong 
magnetic field:

$$
\lambda_{Q C D} \simeq m_{d}\left(\frac{\Lambda_{Q C D}}{\sqrt{|e B|}}\right)^{b / b_{0}}
$$

where $b=\left(11 N_{c}-2 N_{f}\right) / 12 \pi$ and $b_{0}=11 N_{c} / 12 \pi$. Therefore, in a strong magnetic field, $\lambda_{Q C D}$ is much less than $\Lambda_{Q C D}$.

The hierarchy $\lambda_{Q C D} \ll \Lambda_{Q C D}$ is intimately connected with a somewhat puzzling point that the pairing dynamics decouples from the confinement dynamics despite it produces quark masses of order $\Lambda_{Q C D}$ or less [for a magnetic field all the way up to the order of $\left.(10 \mathrm{TeV})^{2}\right]$. The point is that these masses are heavy in units of the new confinement scale $\lambda_{Q C D}$ and the pairing dynamics is indeed weakly coupled.

\section{Arkady question: QCD with a large number of colors}

I did not discuss the case of QCD with a large number of colors in my talk. The question about it was raised by Arkady during the talk (he actually asked not myself, the speaker, but my coauthor Igor Shovkovy who was sitting next to him). Igor and myself got the answer the next day.

Just a look at expression (7) for the gluon mass is enough to recognize that the dynamics in this limit is very different from that considered in the previous sections. Indeed, as is well known, the strong coupling constant $\alpha_{s}$ is proportional to $1 / N_{c}$ in this limit. More precisely, it rescales as $\alpha_{s}=\frac{\tilde{\alpha}_{s}}{N_{c}}$, where the new coupling constant $\tilde{\alpha}_{s}$ remains finite as $N_{c} \rightarrow \infty$ ('t Hooft limit). Then, expression (7) implies that the gluon mass goes to zero in this limit. This in turn implies that the appropriate approximation in this limit is not the improved rainbow approximation but the rainbow approximation itself, when both the vertex and the gluon propagator in the SD equation (4) are taken to be bare. This leads to the following expression for the dynamical mass of quarks [2]:

$$
m_{q}^{2}=C\left|e_{q} B\right| \exp \left[-\pi\left(\frac{\pi N_{c}}{\left(N_{c}^{2}-1\right) \alpha_{s}}\right)^{1 / 2}\right],
$$

where the constant $C$ is of order one. The confinement scale $\lambda_{Q C D}$ is close to $\Lambda_{Q C D}$ in this case [2].

It is natural to ask how large $N_{c}$ should be before the expression (16) becomes reliable. One can show [2] that the threshold value $N_{c}^{t h r}$ grows rapidly with the magnetic field $\left[N_{c}^{t h r} \gtrsim 100\right.$ for $\left.|e B| \gtrsim(1 \mathrm{GeV})^{2}\right]$. Expression (16) for the quark mass is reliable for the values of $N_{c}$ of the order of $N_{c}^{t h r}$ or larger. Decreasing $N_{c}$ below $N_{c}^{t h r}$, one comes to expression (1). 


\section{Conclusion}

QCD in a strong magnetic field yields an example of a rich, sophisticated and (that is very important) controllable dynamics.

\section{Acknowledgments}

I am grateful to the organizers of this Symposium for their warm hospitality. I acknowledge support from the Natural Sciences and Engineering Research Council of Canada.

\section{References}

* On leave of absence from Bogolyubov Institute for Theoretical Physics, 252143, Kiev, Ukraine.

1. V. P. Gusynin, V. A. Miransky, and I. A. Shovkovy, Phys. Rev. Lett. 73, 3499 (1994); Phys. Rev. D 52, 4718 (1995).

2. V. A. Miransky and I. A. Shovkovy, arXiv:hep-ph/0205348 (to appear in Phys. Rev. D).

3. S. Kawati, G. Konisi, and H. Miyata, Phys. Rev. D 28, 1537 (1983); S. P. Klevansky and R. H. Lemmer, Phys. Rev. D 39, 3478 (1989); H. Suganuma and T. Tatsumi, Annals Phys. 208, 470 (1991).

4. S. Schramm, B. Muller, and A. J. Schramm, Mod. Phys. Lett. A 7, 973 (1992).

5. V. P. Gusynin, V. A. Miransky, and I. A. Shovkovy, Phys. Lett. B 349, 477 (1995). Nucl. Phys. B 462, 249 (1996).

6. V. P. Gusynin, V. A. Miransky, and I. A. Shovkovy, Phys. Rev. D 52, 4747 (1995); C. N. Leung, Y. J. Ng, and A. W. Ackley, Phys. Rev. D 54, 4181 (1996); D. S. Lee, C. N. Leung, and Y. J. Ng, Phys. Rev. D 55, 6504 (1997); ibid. D 57, 5224 (1998). D. K. Hong, Y. Kim, and S. J. Sin, Phys. Rev. D 54, 7879 (1996). V. P. Gusynin and A. V. Smilga, Phys. Lett. B 450, 267 (1999). J. Alexandre, K. Farakos and G. Koutsoumbas, Phys. Rev. D 64, 067702 (2001).

7. V. P. Gusynin, V. A. Miransky, and I. A. Shovkovy, Phys. Rev. Lett. 83, 1291 (1999); Nucl. Phys. B 563, 361 (1999).

8. I. A. Shushpanov and A. V. Smilga, Phys. Lett. B 402, 351 (1997); N. O. Agasian and I. A. Shushpanov, Phys. Lett. B 472, 143 (2000). V. C. Zhukovsky, V. V. Khudyakov, K. G. Klimenko, and D. Ebert, JETP Lett. 74, 523 (2001) [Pisma Zh. Eksp. Teor. Fiz. 74, 595 (2001)]; D. Ebert, V. V. Khudyakov, V. C. Zhukovsky, and K. G. Klimenko, Phys. Rev. D 65, 054024 (2002).

9. D. Kabat, K. Lee, and E. Weinberg, Phys. Rev. D 66, 014004 (2002) .

10. J. Schwinger, Phys. Rev. 125, 397 (1962); S. R. Coleman, R. Jackiw, and L. Susskind, Annals Phys. 93, 267 (1975); S. R. Coleman, Annals Phys. 101, 239 (1976). 